\title{
Sex ratios in the rodent malaria parasite, Plasmodium chabaudi
}

\author{
S. E. REECE*, A. B. DUNCAN, S. A. WEST and A. F. READ \\ Institute of Cell, Animal and Population Biology, Ashworth Laboratories, West Mains Road, University of Edinburgh, \\ Edinburgh EH9 $3 \mathcal{Y T}, U K$
}

(Received 30 fanuary 2003; revised 3 fune 2003; accepted 10 fune 2003)

\section{SUMMAR Y}

The sex ratios of malaria and related Apicomplexan parasites play a major role in transmission success. Here, we address 2 fundamental issues in the sex ratios of the rodent malaria parasite, Plasmodium chabaudi. First we test the accuracy of empirical methods for estimating sex ratios in malaria parasites, and show that sex ratios made with standard thin smears may overestimate the proportion of female gametocytes. Secondly, we test whether the mortality rate differs between male and female gametocytes, as assumed by sex ratio theory. Conventional application of sex ratio theory to malaria parasites assumes that the primary sex ratio can be accurately determined from mature gametocytes circulating in the peripheral circulation. We stopped gametocyte production with chloroquine in order to study a cohort of gametocytes in vitro. The mortality rate was significantly higher for female gametocytes, with an average half-life of $8 \mathrm{~h}$ for female gametocytes and $16 \mathrm{~h}$ for male gametocytes.

Key words: sex allocation theory, gametocyte, mortality, blood smears.

\section{INTRODUCTION}

In order for malaria parasites to transmit to new vertebrate hosts, a round of sexual reproduction must be undertaken in the mosquito vector. Sexual stages, termed gametocytes, are produced from the parasite's asexual cycle and are the functional equivalents of males and females. Within 20 min of being taken up in the bloodmeal of a mosquito, the gametocytes have differentiated into gametes (Micks, de Caires \& Franco, 1948; Billker et al. 1997). Each female gametocyte produces 1 female gamete and each male gametocyte can produce up to 8 male gametes (Sinden, 1975; Sinden et al. 1978; Janse et al. 1989; Schall, 2000). Male gametes are motile (each has a flagellum), and can fertilize female gametes either from the same clonal lineage (genotype), or outcross with other genotypes. The fertilized zygotes undergo several stages of asexual replication before migrating to their vector's mouthparts, ready to infect a new host. The gametocyte sex ratio (defined as the proportion of male gametocytes) is an important factor in determining how well a parasite genotype maximizes its genetic representation in the population of new infections (Robert et al. 1996; Paul et al. 2000; Schall, 2000; Paul, Brey \& Robert, 2002). Recently there has been an increased interest in using evolutionary theory to explain the sex ratios observed in malaria parasites (reviewed by West, Reece $\&$ Read, 2001; Read et al. 2002). Theory predicts that the sex

\footnotetext{
* Corresponding author. Tel: +44 131650 6468. Fax: +44131650 6465. E-mail: sarah.reece@ed.ac.uk
}

ratio $\left(r^{*}\right)$, should be related to the inbreeding rate by the equation $r^{*}=(1-F) / 2$, where $F$ is Wrights coefficient of inbreeding (the probability that 2 homologous genes in 2 mating gametes are identical by descent; Dye \& Godfray, 1993 ; West, Smith \& Read, 2000; Nee, West \& Read, 2002). Whilst this relationship has enabled a broad-scale understanding of the sex ratios observed in malaria and related Apicomplexan parasites, there are discrepancies that demand elucidation (West et al. 2001; Paul et al. 2002). To date, there has been little work on how appropriate standard empirical measurements of apicomplexan sex ratios are for sex allocation theory (Read et al. 2002). Here, we address this issue.

First, we tested the accuracy of standard methods for estimating gametocyte sex ratios. The sex of gametocytes is usually assigned by examination of thin blood smears stained with Giemsa's solution. However, this method may overestimate the proportion of female gametocytes if maturing male gametocytes resemble females and are sexed wrongly from thin blood smears (Schall, 1989; Dearsly, Sinden \& Self, 1990). Sex ratio estimates made with specific molecular markers can be significantly less female biased than when made with thin blood smears although it should be noted that no concurrent comparison has been made (Ranford-Cartwright et al. 1993; Smith et al. 2000, but see Silvestrini, Alano \& Williams, 2000). In addition, sex ratio estimates can be much more female biased than expected - in extreme cases there can not be enough males to fertilize all of the female gametes (Burkot, Williams \& Schneider, 1984; Pickering et al. 2000). We tested 
this possibility using the rodent malaria parasite Plasmodium chabaudi, by comparing the sex ratios observed in thin blood smears with sex ratios observed using a method in which gametocytes are partially allowed to differentiate into gametes to reveal their sex more clearly.

Secondly, we tested the assumption that the mortality rate of male and female gametocytes is equal in $P$. chabaudi. Sex ratio theory is concerned with predicting the sex ratio at the point when sexual differentiation occurs (defined as the primary sex ratio), and differential mortality results in the observed sex ratio (the secondary sex ratio), differing from the primary sex ratio. Primary and secondary sex ratios may differ for a number of non-exclusive reasons. In most organisms, females live longer than males (Owens, 2002). For malaria parasites, sex-biased mortality could occur in a number of ways. Male and female sex-specific antigens exist (Severini et al. 1999; Eksi \& Williamson, 2002), so that male and female gametocytes could be killed by host immunity at different rates (Paul et al. 2000, 2002; Reece \& Read, 2000). In species that sequester there may be sex specific rates for sequestration in the capillaries. If the mortality rate is greater for male gametocytes, natural selection could favour a less female biased sex ratio for a given level of inbreeding to insure there are enough male gametes in the bloodmeal to fertilize all the female gametes (Paul et al. 2000, 2002; West et al. 2001, 2002; Gardner, Reece \& West, 2003).

\section{MATERIALS AND METHODS}

\section{Parasites and hosts}

We gave 11-week-old female C57 black mice (Harlan-Olac, UK) an intraperitoneal inoculation of $10^{6}$ red blood cells parasitized with Plasmodium chabaudi, clone ER (WHO Registry of Standard Malaria Parasites, University of Edinburgh, UK). We administered parasites in $0 \cdot 1 \mathrm{ml}$ doses consisting of $47 \cdot 5 \%$ Ringers ( $27 \mathrm{~mm} \mathrm{KCl}, 27 \mathrm{mM} \mathrm{CaCl}_{2}, 0 \cdot 15 \mathrm{M}$ $\mathrm{NaCl}$ ), $50 \%$ heat-inactivated calf serum and $2 \cdot 5 \%$ heparin (200 units $/ \mathrm{ml}$ ). We housed all mice in groups of 5 at $20{ }^{\circ} \mathrm{C}$ with a $12 \mathrm{~h}$ light $/ 12 \mathrm{~h}$ dark cycle. We provided food (41B, Harlan-Teklad, UK) and water with $0.05 \%$ PABA (to enhance parasite growth) ad libitum.

\section{Comparison of methods for sexing gametocytes}

We took blood samples from the tail veins of 5 mice on 6 occasions during days 15-17 post-infection (p.i.). For each mouse at each sampling point, we made 3 blood smears immediately ('immediate' smears), and 3 smears after $15 \mathrm{~min}$ when gametocytes began differentiation ('delayed' smears). We recorded the total numbers of male and female gametocytes in the 3 smears from each sampling point for each method. We only sexed gametocytes that were large enough to be easily differentiated from all asexual stages in order to avoid problems associated with identifying and sexing young gametocytes. We made the delayed smears according to the following protocol. Approximately $1 \mu \mathrm{l}$ of blood was taken from the tail vein and kept in the cap of a $0.5 \mathrm{ml}$ Eppendorf tube containing warm water (humidity prevents blood clotting), and the cooling blood was dropped onto 3 slides after $15 \mathrm{~min}$, using a $20 \mu \mathrm{l}$ pipette and then smeared. All smears were fixed in absolute methanol and stained with $10 \%$ Giemsa's solution.

For each 'immediate' smear, we sexed gametocytes according to published criteria: (1) the cytoplasm of males is pink and that of females a pale blue; (2) males tend to be crescent shaped and females ring shaped and (3) females have a compact nucleus with a vacuole beside it and the nucleus in males is dispersed with a pale rim around it (Carter $\&$ Walliker, 1975; Landau \& Boulard, 1978; Sinden et al. 1978; Schall, 1989; Dearsly et al. 1990). In contrast, gametocytes in 'delayed' smears had begun to differentiate into gametes and take on a different morphology. Gametocytes require a $5{ }^{\circ} \mathrm{C}$ drop in temperature and an increase in $\mathrm{pH}$ to become activated (Billker et al. 1997; Ogwan'g et al. 1993). The temperature drop was achieved as soon as blood was removed from the tail vein and the $\mathrm{pH}$ increased as $\mathrm{CO}_{2}$ diffused out of the blood. Female gametes remained in their red blood cell and appeared as described above, or had burst out and 'rounded up,' where they condense into a circular shape, and stain a more intense blue (Sinden, 1975, 1983; Sinden et al. 1978). Males undergo rapid DNA proliferation and, as their nucleus expands, the cytoplasm becomes pushed away and forms a ring around the large circular nucleus (Kawamoto et al. 1992). After 15$20 \mathrm{~min}$ the male gametocyte begins to package up DNA into separate flagella which extend from the nucleus and stain red-purple (Janse et al. 1986; Kawamoto et al. 1992). Male gametocytes eventually detach and swim off to locate female gametes but, at least in vitro, this took longer than the $15 \mathrm{~min}$ allowed by our protocol.

\section{Differential mortality experiment}

Each mouse in the treatment $(\mathrm{CQ})$ group was given a curative dose of chloroquine sulphate (Nivaquine ${ }^{\mathrm{TM}}$ ) dissolved in distilled water, and mice in the control group mice received distilled water. Conventional wisdom states that curative chloroquine treatment kills all asexuals and very young gametes, leaving an infection composed of mature gametocytes (Peters, 1970). Sex-specific clearance of gametocytes can then be measured directly (Smalley \& Sinden, 1977). A trial with 6 mice showed that a dose of $30 \mathrm{mg} / \mathrm{kg}$ clears asexual parasites to below detectable levels, for at least 2 days post-administration (asexual density $\times 10^{9} / \mathrm{ml}$ prior to treatment, $0 \cdot 86 \pm 0 \cdot 35$; after 
$24 \mathrm{~h}, 0 \cdot 03 \pm 0 \cdot 01$; after 48 and $72 \mathrm{~h}$, no parasites observed). We administered drugs and placebos at $08.00 \mathrm{~h}$ on day 14 p.i., when peak gametocytaemia is expected, and then sampled each mouse throughout days 14 and 15 p.i. P. chabaudi gametocytes are thought to be produced synchronously at midnight, and therefore, in this experiment we monitored the 2 cohorts of gametocytes produced over the 2 days prior to drug treatment.

To collect sex ratio data, we made smears following the method detailed above for 'delayed smears' for each mouse every $2.5 \mathrm{~h}$ on both days, from $08.30 \mathrm{~h}$ to $21.00 \mathrm{~h}$. We calculated gametocyte density from the product of red blood cell densities and gametocytaemia (proportion of red blood cells infected with gametocytes). We made thin smears every $5 \mathrm{~h}$ (i.e. every other sampling point) for the first $36 \mathrm{~h}$ and at 51 and $75 \mathrm{~h}$ post-treatment. We stained all smears using a $10 \%$ Giemsa's stain buffered in $90 \%$ phosphate solution for $10 \mathrm{~min}$.

\section{Analysis}

For both experiments we carried out the data analysis using S-Plus (Insightful Corporation). We compared the methods for sexing gametocytes using a paired $t$-test, by arcsin square root transforming the sex ratios to allow the assumption of normal errors. For the differential mortality data, gametocyte densities decreased exponentially so we logarithmically transformed the data as $\ln$ (gametocyte density $+0 \cdot 001$ ). To calculate the life-span of gametocytes we considered the gametocyte density data from the chloroquine group only. If we assume a constant mortality rate $b$ (number of gametocytes $\times 10^{6} / \mathrm{ml}$ ), and initial gametocyte density $a$, the density at time $x$ equals $y$; given by: $y=a e^{-b x}$. Taking the natural $\log$ of both sides gives $\ln (y)=\ln (a)-b x$, therefore the regression of time against $\ln$ (gametocyte density) provides the mortality rate ( $b$ : the slope) and initial gametocyte density ( $a$ : the intercept) for each mouse. Further analyses were then carried out using the regression data for each mouse to avoid pseudoreplication. From these mortality rates we calculated the halflife of gametocytes in each mouse $\left(T_{1 / 2}\right.$; time required for the gametocyte density to decrease to half of the initial density) using the function: $T_{1 / 2}=$ $[-\ln (1 / 2)] / b$.

\section{RESULTS}

\section{Comparison of methods for sexing gametocytes}

The mean ( \pm s.E.) number of gametocytes sexed in each smear was $52 \cdot 26( \pm 2 \cdot 88)$ and we analysed 28 pairs of samples. Sex ratios determined from smears made immediately were significantly more female biased than those determined from delayed smears (Fig. 1; mean difference \pm s.E. $=0 \cdot 12 \pm 0 \cdot 03, n=28$, paired $t$-test, $T=4.92, P<0 \cdot 001)$.

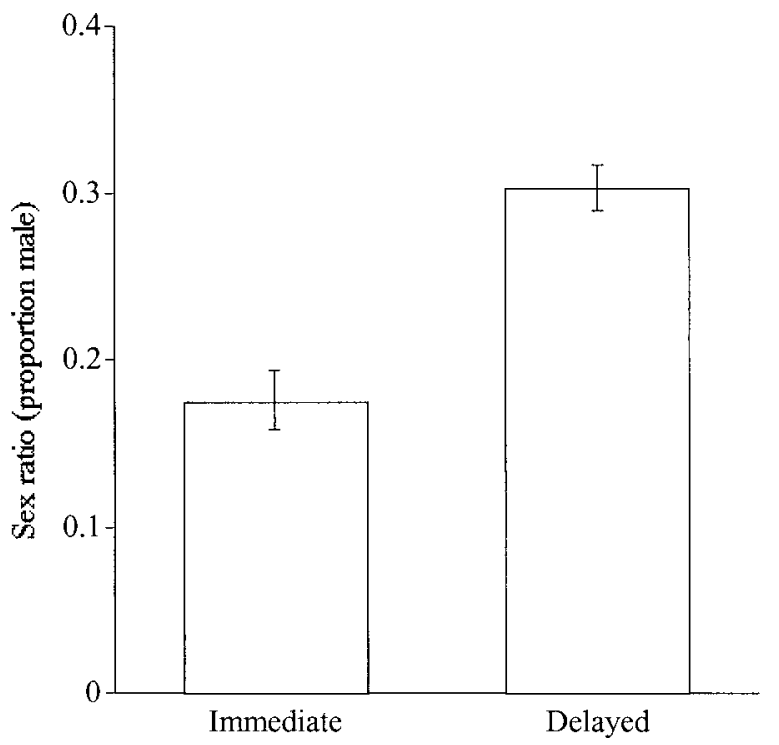

Fig. 1. Mean sex ratio estimated from thin blood smears made with blood immediately after being taken from the tail vein (immediate), and smears made from blood in which gametogenesis had begun (delayed). Bars are the standard error of the mean, $n=28$ pairs.

\section{Differential mortality experiment}

The gametocyte density $\left(\times 10^{6} / \mathrm{ml}\right)$ of the chloroquine group decreased throughout the sampling period at a significantly faster rate than the control group (Fig. 2; mean \pm s.E. slope for $\mathrm{CQ}=-0.02 \pm$ $0 \cdot 001 ;$ mean \pm s.E. for control $=-0 \cdot 008 \pm 0 \cdot 0008$; $n=16, T=4 \cdot 16, P=0 \cdot 001)$. In the chloroquine group asexuals were not observed post-treatment. Thus, the drug treatment worked: asexuals were killed and surviving gametocytes died without replacement over the subsequent days. There was no significant difference in red blood cell densities of the 2 groups throughout the sampling period (mean slope \pm s.E. for $\mathrm{CQ}=-0 \cdot 01 \pm 0 \cdot 003$; mean slope \pm s.E. for control $=-0 \cdot 01 \pm 0 \cdot 006 ; n=20, T=0 \cdot 98, P=0 \cdot 34)$.

The mean gametocyte half-life was $14 \mathrm{~h}(95 \%$ C.I. 11-21 h). The density of female gametocytes decreased significantly faster than male gametocytes (mean mortality rate, $b \pm$ s.E., for females $=0.09 \pm$ $0 \cdot 009, n=10$; mean for males $=0 \cdot 04 \pm 0 \cdot 01, n=9$; paired $t$-test, $T=3.94, n=9$ pairs, $P=0 \cdot 004)$. The half-life for female gametocytes was $8 \mathrm{~h}(95 \%$ C.I. $5-13 \mathrm{~h})$, whereas the half-life for males was $16 \mathrm{~h}$ (95\% C.I. 10-41 h). That is, female gametocytes were lost from the circulation twice as fast as males (Fig. 3).

\section{Comparison of primary and secondary sex ratios}

We estimated the consequences of this differential mortality for how much observed (secondary) sex ratios will differ from the sex ratio at gametocyte maturity (primary sex ratio). Although a cohort of parasites destined to become gametocytes will be 

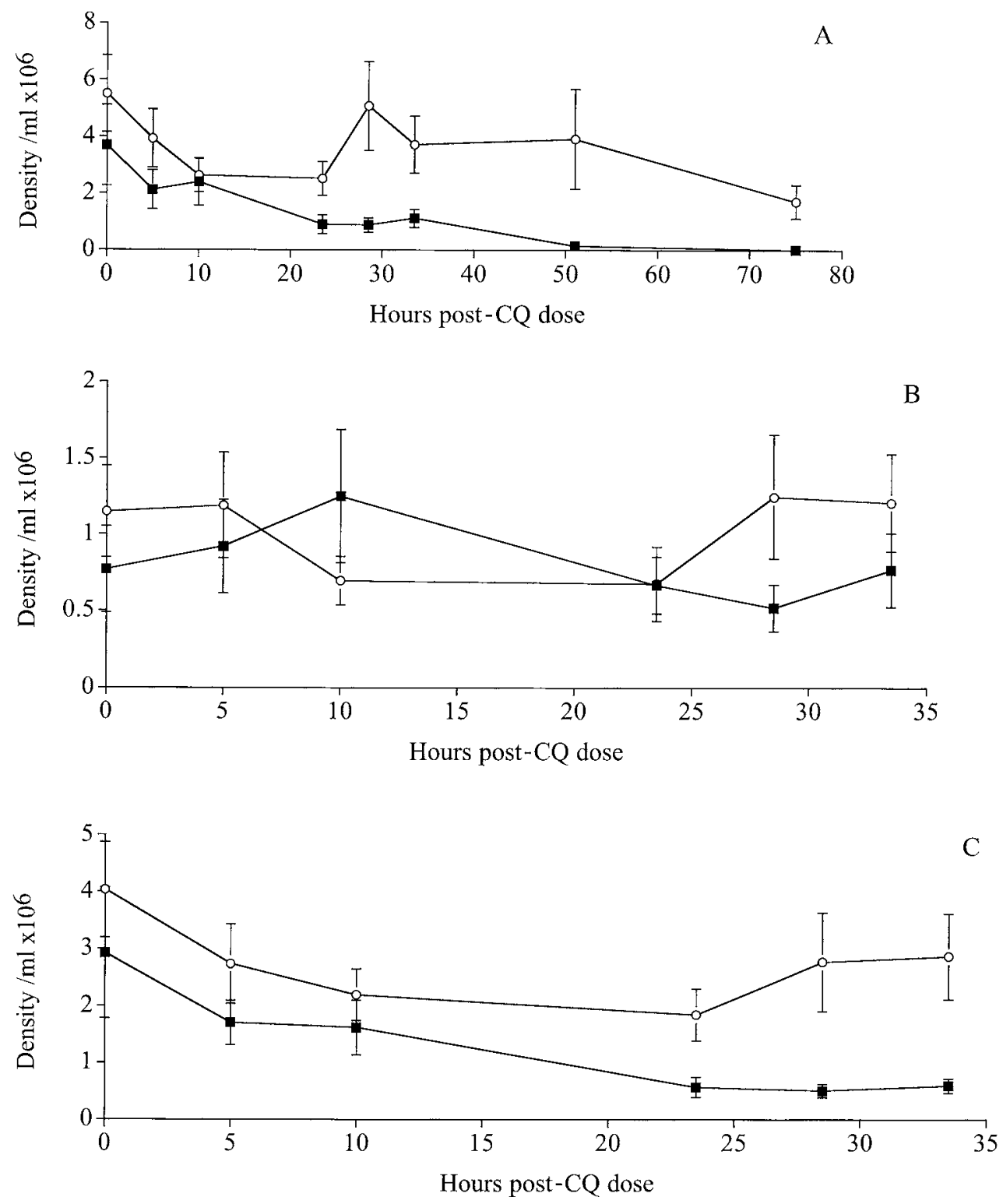

Fig. 2. (A) Total, (B) male and (C) female gametocyte density throughout the sampling period in infections treated with chloroquine $(\boldsymbol{\square})$ or distilled water $(\bigcirc)$. The density in the chloroquine group decreased at a significantly faster rate than the control group.

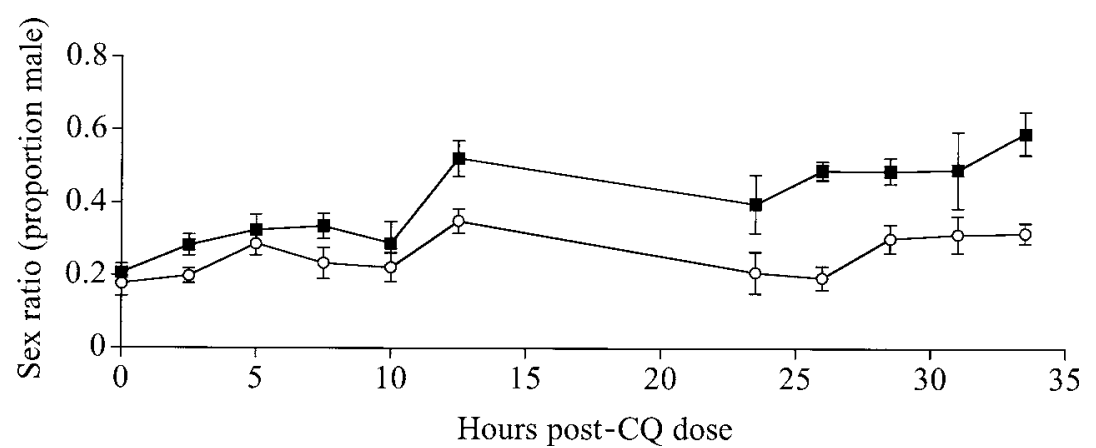

Fig. 3. Sex ratio of the chloroquine group $(\boldsymbol{\square})$ became less female biased over the sampling period than the control group $(\bigcirc)$.

initiated synchronously at schizogony, there are 2 scenario extremes for their recruitment as mature gametocytes: they mature at the same time (complete synchrony) or they have variable maturation times leading to continuous recruitment (asynchrony). We consider these 2 scenarios separately but reality is likely to lie between these extremes. Assuming recruitment is continuous, the initial density of each 


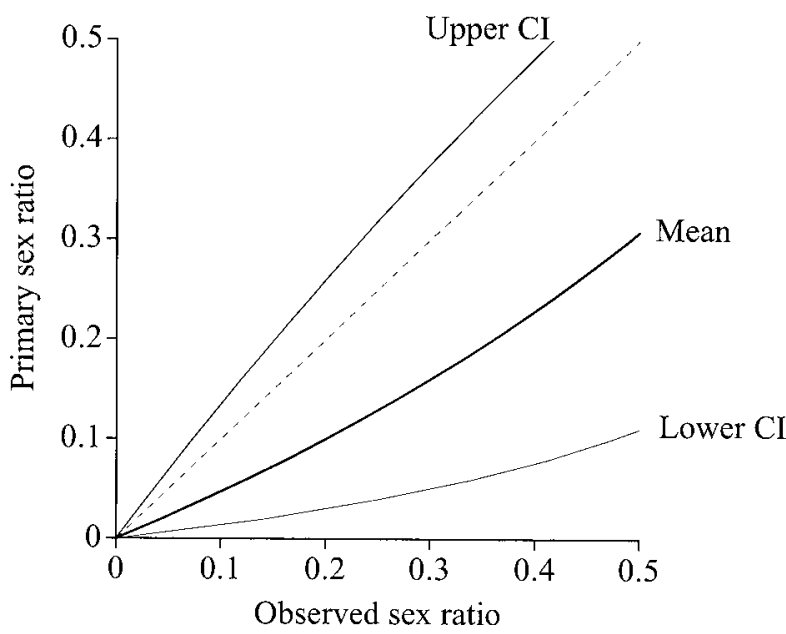

Fig. 4. For asynchronous gametocyte production. The unbroken lines show the primary sex ratio (before mortaility), for a range of observed (secondary) sex ratios and the $95 \%$ confidence intervals for this relationship. The dashed line is $y=x$, i.e. when the primary and secondary sex ratios are equal.

sex can be calculated for time $x$ from: Initial $=$ $\int_{0}^{\infty} c e^{-b x} d x$, where $c$ is the sex-specific recruitment value and $b$, the sex-specific mortality rate. Integrating this function and incorporating the observed sex ratio $r$, gives an equation for the initial density of each sex: Male $=r c / b_{\text {male }}$ and Female $=$ $[c(r-1)] / b_{\text {female }}$ and the initial sex ratio is given by: $R=r / b_{\text {male }} /\left[r / b_{\text {male }}+(1-r) / b_{\text {female }}\right]$. Figure 4 shows the difference between the observed and primary sex ratio. The average sex ratio observed in the control group was $0 \cdot 2$, which corresponds to a primary sex ratio of $0 \cdot 1(95 \%$ C.I. $0 \cdot 03-0 \cdot 26)$.

If we assume all gametocytes mature synchronously, e.g. at schizogony $(24.00 \mathrm{~h})$, using the same logic as above, the primary sex ratio $R$, can be established from the function: $R=r e^{-t b_{\text {male }}} /\left[r e^{-t b_{\text {male }}}+\right.$ $\left.(1-r) e^{-t b_{\text {female }}}\right]$, where $r$ is the observed sex ratio, $t$ the time since maturation and $b$ the sex-specific mortality rates. Figure 5 shows the discrepancy between observed and primary sex ratio for synchronous recruitment at several different sampling points. The discrepancy between primary and secondary sex ratios increases with time since recruitment, for example, for a sex ratio of $0 \cdot 2$, observed $12 \mathrm{~h}$ after recruitment, the primary sex ratio is $0.12(95 \%$ C.I. $0 \cdot 07-0 \cdot 24)$.

\section{DISCUSSION}

Our first experiment demonstrates that sex ratios estimated from standard blood smears may overestimate the proportion of gametocytes that are female. Our second experiment shows that in $P$. chabaudi, female gametocytes were lost from the circulation approximately twice as fast as males (halflives of 8 and $16 \mathrm{~h}$ respectively).

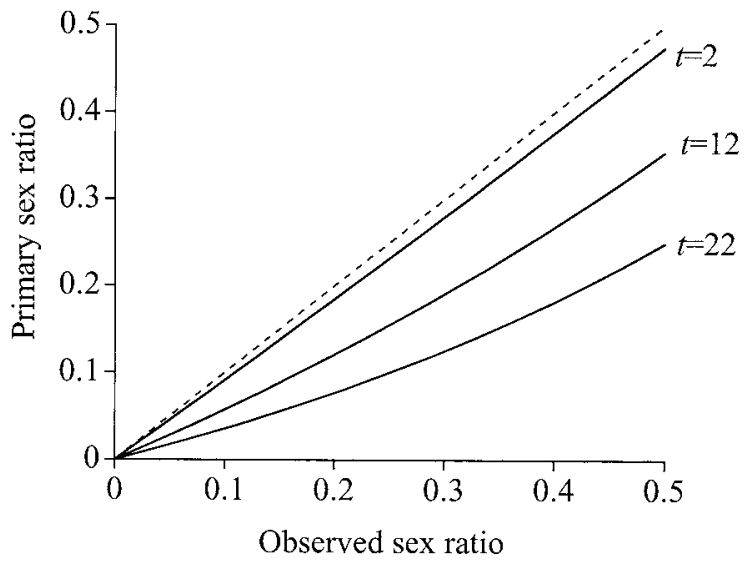

Fig. 5. The relationship between the primary sex ratio (before mortaility), for a range of observed (secondary) sex ratios for 3 times $(t \mathrm{~h})$ since synchronous gametocyte production. The dashed line is $y=x$, i.e. when the primary and secondary sex ratios are equal or when $t=0 \mathrm{~h}$.

\section{Comparison of methods for sexing gametocytes}

Our results suggest that standard methods for obtaining sex ratios may lead to an over-estimation of the extent of female bias in P. chabaudi gametocytes. It is probable that male gametocytes were misidentified as females in smears made immediately from fresh blood (Schall, 1989; Dearsly et al. 1990; Smith et al. 2000). Giemsa's stain stains female gametocytes blue because they are rich in basophilic structures (e.g. ribosomes and endoplasmic reticulum). If young mature male gametocytes are rich in ribosomes there may be a period when males resemble females and can begin gametogenesis (Sinden, 1975; Sinden, Canning \& Spain, 1976; Aikawa et al. 1981). Males may lose their ribosomes on maturation if they are not required for gamete production, but female gametes may need to retain them for zygote development. If gametocytes of other Plasmodium species share similar developmental pathways to $P$. chabaudi, then males may resemble females for a period of their time in circulation and lead to biased sex ratio estimates (RanfordCartwright et al. 1993). A less plausible explanation for our results is that factors such as macrophages are selectively attacking extracellular female gametocytes in delayed smears. We believe that this is unlikely because there is no evidence that males or macrophages are preferentially attracted to females. Moreover, the activity of macrophages is likely to be severely restricted in rapidly cooling and clotting blood.

\section{Differential mortality and life-span of gametocytes}

The discovery that female gametocytes are lost from the circulation at a faster rate than male gametocytes was unexpected. Female gametocytes could be lost 
from the circulation at a faster rate because they are larger at maturity and perhaps have a greater tendency than males to get 'stuck' in peripheral blood vessels (Fallis \& Desser, 1974; Schall, 1989; Bennett \& Peirce, 1992, but see Read et al. 1992; Smith et al. 2000). Alternatively, they could be more antigenic or vulnerable to immune killing if they are more metabolically active (Severini et al. 1999; Eksi \& Williamson, 2002). Our mortality rate estimates show that the observed sex ratio may differ from the primary sex ratio whether gametocyte recruitment is synchronous or asynchronous. If gametocyte recruitment is asynchronous the primary sex ratio is approximately half the observed sex ratio whereas the discrepancy increases with time since gametocyte production for synchronous recruitment. As recruitment of gametocytes is thought to be largely synchronous, sampling close to schizogony should minimize this discrepancy.

An alternative explanation for our results is that chloroquine could exert different effects on male and female gametocytes. Chloroquine could only affect female gametocytes more than males if they metabolise haematin for a longer period. This may seem unlikely, but is yet to be investigated. However, a study using pyrimethamine sulphadoxine to estimate sex-specific half-lives in Plasmodium falciparum produced qualitatively similar results to those presented here (Sowunmi et al. unpublished observations). Pyrimethamine sulphadoxine prevents DNA replication and thus could not have different effects on male and female gametocytes whilst in the host (Peters, 1970). Furthermore, if chloroquine killed all asexuals and metabolizing gametocytes and then is rapidly cleared by the host (the half-life is $<20 \mathrm{~h}$; Macomber, O'Brien \& Hahn, 1966) then we were tracking a cohort of gametocytes whose survival is less likely to have been affected by the drug.

These data raise several issues worthy of investigation. Does differential mortality also occur at the start of an infection, before host immunity appears? Determining this would go some way to establishing whether faster clearance of female gametocytes is due to host or intrinsic factors. Does greater female mortality significantly reduce the numbers of viable female gametes in the vector? Recent thinking has shown that parasites may need to invest more in males than predicted by their population structure in order to fertilize all female gametes (Paul et al. 2000, 2002; West, Smith \& Read, 2002; Gardner et al. 2003); perhaps higher female mortality renders this unimportant. Finally, sex ratios in several Plasmodium species become less female biased as infections progress, and a variety of ideas have been suggested to account for this (Paul et al. 2000, 2002). Could an accumulation of longer-lived male gametocytes account for these increases in sex ratio?
We would like to thank A. Graham for help with the experimental work, staff at the March animal house, the NERC, BBSRC, Royal Society and Wellcome Trust for financial support and J. DeRoode for discussion.

\section{REFERENCES}

AikaWA, M., RENER, J., CARTER, R. \& Miller, L. H. (1981). An electron microscopical study of the interaction of monoclonal antibodies with gametes of the malaria parasite Plasmodium gallinaceum. Fournal of Protozoology 28, 383-388.

BENNETT, G. F. \& PEIRCE, M. A. (1992). Leucocytozoids of 7 old world passeriform families. Fournal of Natural History 26, 693-707.

BILlKer, O., SHAW, M. K., MARGOS, G. \& Sinden, R. E. (1997). The roles of temperature, $\mathrm{pH}$ and mosquito factors as triggers of male and female gametogenesis of Plasmodium berghei in vitro. Parasitology 114, 1-7.

BURKOT, T., WILLIAMS, J. L. \& SCHNEIDER, I. (1984). Infectivity to mosquitoes of Plasmodium falciparum clones grown in vitro from the same isolate. Transactions of the Royal Society of Tropical Medicine and Hygiene $\mathbf{7 8}$, 339-341.

CARTER, R. \& WALliker, D. (1975). New observations on the malaria parasites of rodents of the Central African Republic - Plasmodium vinckei petteri subsp. nov. and Plasmodium chabaudi Landau, 1965. Annals of Tropical Medicine and Parasitology 69, 187-196

DEARSLy, A. L., Sinden, R. E. \& SELF, I. A. (1990). Sexual development in malarial parasites: gametocyte production, fertility and infectivity to the mosquito vector. Parasitology 3, 359-368.

DYE, C. \& GODFRAY, H. J. C. (1993). On sex ratio and inbreeding in malaria parasite populations. Fournal of Theoretical Biology 161, 131-134.

EKsi, s. \& Williamson, K. C. (2002). Male-specific expression of the paralog of malaria transmission blocking target antigen Pfs230, PfB0400w. Molecular and Biochemical Parasitology 122, 127-130.

FALlis, A. M. \& DESSER, S. S. (1974). On species of Leucocytozoan. Advances in Parasitology 12, 1-67.

GARDNER, A., REECE, S. E. \& WEST, S. A. (2003). Even more extreme fertility insurance and the sex ratios of protozoan blood parasites. Fournal of Theoretical Biology (in the Press).

JANSE, C. J., BOORSMA, E. G., RAMESAR, J., VANVIANEN, P., VANDERMEER, R., ZENOBI, P., CASAGLIA, O., MONS, B. \& VAnderberg, F. M. (1989). Plasmodium bergheigametocyte production, DNA content, and chromosome-size polymorphisms during asexual multiplication in vivo. Experimental Parasitology 68, 274-282.

JANSE, C. J., VANDERKLOOSTER, P. F. J., VANDERKAAY, H. J., VANDERPLOEG, M. \& OVERDUlVE, J. P. (1986). Rapid repeated DNA-replication during microgametogenesis and DNA-synthesis in young zygotes of Plasmodium berghei. Transactions of the Royal Society of Tropical Medicine and Hygiene 80, 154-157.

KAWAMOTO, F., KIDO, N., HANAICHI, T., DJAMGOZ, M. B. A. \& SINDEN, R. E. (1992). Gamete development in Plasmodium berghei regulated by ionic exchange mechanisms. Parasitology Research 78, 277-284. 
LANDAU, I. \& BOULARD, Y. (1978). Life cycles and morphology. In Rodent Malaria (ed. Killick-Kendrick, R. \& Peters, W.), pp. 53-84. Academic Press, London. MACOMBER, P. B., O'BRIEN, R. L. \& HAHN, F. E. (1966). Chloroquine: physiological basis of drug resistance in Plasmodium berghei. Science 152, 1374-1375.

Micks, D. W., De CAIREs, P. F. \& FRANCO, L. B. (1948). The relationship of exflagellation in avian Plasmodia to $\mathrm{pH}$ and immunity in the mosquito. American Fournal of Hygiene 48, 182-190.

NEE, S., WEST, S. A. \& READ, A. F. (2002). Inbreeding and parasite sex ratios. Proceedings of the Royal Society of London, Series B 269, 755-760.

OGWAN'G, R. A., MWANGi, J. K., GITHURE, J., WERE, J. B. O., ROBERTS, C. R. \& MARTIN, S. K. (1993). Factors affecting exflagellation of in vitro cultivated Plasmodium falciparum gametocytes. American Journal of Tropical Medicine and Hygiene 49, 25-29.

owENs, I. P. F. (2002). Sex differences in mortality rate. Science 297, 2008-2009.

PAUl, R. E. L., Brey, P. T. \& ROBERT, v. (2002). Plasmodium sex determination and transmission to mosquitoes. Trends in Parasitology 18, 32-38.

PAUl, R. E. L., COULSON, T. N., RAIBAUD, A. \& BREY, P. T. (2000). Sex determination in malaria parasites. Science 287, 128-131.

PETERs, w. (1970). The pharmacology and mode of action of antimalarial drugs with especial reference to drug resistance. In Chemotherapy and Drug resistance in Malaria. Academic Press, London.

PICKERING, J., READ, A. F., GUERrERo, S. \& WEST, s. A. (2000). Sex ratio and virulence in two species of lizard malaria parasites. Evolutionary Ecology Research 2, 171-184.

RANFORD-CARTWRIGHT, L. C., BALFE, P., CARTER, R. \& WALLIKER, D. (1993). Frequency of cross fertilisation in the human malaria parasite Plasmodium falciparum. Parasitology 107, 11-18.

READ, A. F., NARARA, A., NEE, S., KEYMER, A. E. \& DAY, K. P. (1992). Gametocyte sex ratios as indirect measures of outcrossing rates in malaria. Parasitology 104, 387-395.

READ, A. F., SMith, T. G., NEE, S. \& WEST, S. A. (2002). Sex ratios of malaria parasites and related protozoa. In Sex Ratios: Concepts and Research Methods (ed. Hardy, I. C. W.), pp. 314-332. Cambridge University Press, Cambridge.

REECE, S. E. \& READ, A. (2000). Malaria sex ratios. Trends in Ecology and Evolution 15, 259-260.

ROBERT, V., READ, A. F., ESSONG, J., TCHUINKAM, T., MULDER, B., verhave, J. P. \& CARNEVAlE, P. (1996). Effect of gametocyte sex ratio on infectivity of Plasmodium falciparum to Anopheles gambiae. Transactions of the
Royal Society of Tropical Medicine and Hygiene 90, 621-624.

Schall, J. J. (1989). The sex ratio of Plasmodium gametocytes. Parasitology 98, 343-350.

Schall, J. J. (2000). Transmission success of the malaria parasite Plasmodium mexicanum into its vector: role of gametocyte density and sex ratio. Parasitology 121, 575-580.

SEVERINI, C., SILVESTRINI, F., SANNELla, A., BARCA, S., GRADONI, L. \& ALANO, P. (1999). The production of the osmiophillic body protein Pfg 377 is associated with state of maturation and sex in Plasmodium falciparum gametocytes. Molecular and Biochemical Parasitology 100, 247-252.

Silvestrini, F., AlANO, P. \& Williams, J. L. (2000). Commitment to the production of male and female gametocytes in the human malaria parasite Plasmodium falciparum. Parasitology 121, 465-471.

SINDEN, R. E. (1975). Microgametogenesis in Plasmodium yoeli nigeriensis: a scanning electron microscope investigation. Protistologica 11, 263-268.

SINDEN, R. E. (1983). Sexual development of malarial parasites. Advances in Parasitology 22, 153-216.

SINDEN, R. E., CANNING, E. U., BRAY, R. S. \& SMALlEY, M. E. (1978). Gametocyte and gamete development in Plasmodium falciparum. Proceedings of the Royal Society of London, Series B 201, 375-399.

SINDEN, R. E., CANNING, E. U. \& SPAIN, B. (1976). Gametogenesis and fertilisation in Plasmodium yoeli nigeriensis: a transmission electron microscope study. Proceedings of the Royal Society of London, Series B 193, 55-76.

SMAlley, M. E. \& SINDEN, R. E. (1977). Plasmodium falciparum gametocytes: their longevity and infectivity. Parasitology 74, 1-8.

SMith, T. G., LOURENCO, P., CARTER, R., WALliker, D. \& RANFORD-CARTwRIGHT, L. (2000). Commitment to sexual differentiation in the human malaria parasite Plasmodium falciparum. Parasitology 121, 127-133.

WEST, S. A., REECE, S. E. \& READ, A. F. (2001). The evolution of gametocyte sex ratios in malaria and related apicomplexan (protozoan) parasites. Trends in Parasitology 17, 525-531.

WEst, s. A., SMith, T. G. \& READ, A. F. (2000). Sex allocation and population structure in apicomplexan (protozoa) parasites. Proceedings of the Royal Society of London, Series B 267, 257-263.

WEST, S. A., SMITH, T. G. \& READ, A. F. (2002). Fertility insurance and the sex ratios of malaria and related Hemosporin blood parasites. Fournal of Parasitology 88, 258-263. 